\title{
The frequency and characteristics of idiopathic osteosclerosis and condensing osteitis lesions in a Turkish patient population
}

\author{
Ozkan Miloglu ${ }^{1}$, Ertan Yalcin ${ }^{2}$, Mustafa-Cemil Buyukkurt ${ }^{3}$, Hamit Acemoglu ${ }^{4}$
}

\author{
${ }^{1}$ Research Assistant. Department of Oral Diagnosis and Oral Radiology. Faculty of Dentistry, Ataturk University, Erzurum, \\ Turkey \\ ${ }^{2}$ Research Assistant. Department of Oral and Maxillofacial Surgery. Faculty of Dentistry, Ataturk University, Erzurum, Turkey \\ ${ }^{3}$ Associated Professor. Department of Oral and Maxillofacial Surgery. Faculty of Dentistry, Ataturk University, Erzurum, Turkey \\ ${ }^{4}$ Associated Professor. Department of Medical Education. Faculty of Medicine, Ataturk University, Erzurum, Turkey
}

Correspondence:

Ataturk University

Faculty of Dentistry,

Department of Oral Diagnosis and Radiology,

25240, Erzurum, TURKEY

omiloglu@hotmail.com

Received: 27/02/2009

Accepted: 08/06/2009
Miloglu O, Yalcin E, Buyukkurt MC, Acemoglu H. The frequency and characteristics of idiopathic osteosclerosis and condensing osteitis lesions in a Turkish patient population. Med Oral Patol Oral Cir Bucal. 2009 Dec 1:14 (12):e640-5.

http://www.medicinaoral.com/medoralfree01/v14i12/medoralv14i12p640.pdf

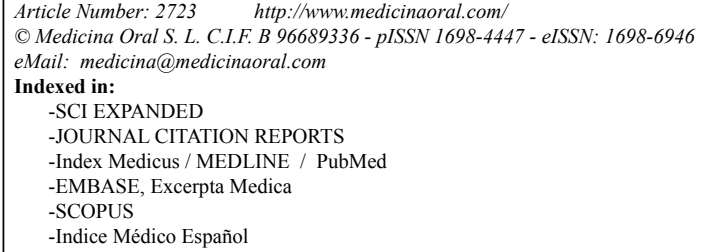

\begin{abstract}
Objectives: The objective of this study was to investigate the frequencies of idiopathic osteosclerosis (IO) and condensing osteitis (CO) in a Turkish patient population with respect to age and sex, in addition to shape, localization, and the dental relationship of $\mathrm{IO}$ and $\mathrm{CO}$ lesions.

Patients and methods: A retrospective study was performed using panoramic radiographs of 6,154 patients ranging in age from 5 to 69 subjected to dental treatment. Descriptive characteristics of radiopacities, including shape, localization, and dental relationship were recorded. The Chi-squared test was used.

Results: There were 238 radiopacities detected, 185 IO lesions in 150 (2.44\%) subjects (96 female, 54 male and mean age: 26.2), and $53 \mathrm{CO}$ lesions in $50(0.81 \%)$ subjects ( 27 female, 23 male and mean age: 32.8$)$. Both IO and $\mathrm{CO}$ lesions were found to be higher in number among females when compared to males. However, this difference was statistically significant only for IO lesions $(p<0.001)$. The frequency of IO lesions was found to be significantly higher in the 3rd and 4 th decades of life $(20-39$ years) than in other decades $(p<0.001)$. On the other hand, the frequency of $\mathrm{CO}$ lesions was the similar in the age ranges of 20-39 years and 40-69 years, and its frequency in these periods was statistically higher than in the 1st and 2nd decades of life $(\mathrm{p}<0.01)$.

Conclusion: Our results point to the low IO and CO frequency among the Turkish population. In addition, our findings support the theory that IO lesions are developmental variations of normal bone architecture unrelated to a local stimulant and $\mathrm{CO}$ lesions could be considered reactive formations related to teeth with severe caries, restoration, or pulpitis.
\end{abstract}

Key words: Frequency, idiopathic osteosclerosis, condensing osteitis, orthopantomography, Turkish. 


\section{Introduction}

Idiopathic osteosclerosis (IO) refers to a focal area of increased radiodensity that is of unknown cause and cannot be attributed to any inflammatory, dysplastic, neoplasia, or systemic disorder. IO is invariably asymptomatic, not associated with detectable cortical expansion, and is typically detected during a routine radiographic examination (1). IO has also been termed dense bone island, bone eburnation, bone whorl, bone scar, enostosis, and focal periapical osteopetrosis (1-6). Similar radiopaque foci may develop in the periapical areas of teeth with nonvital or significantly inflamed pulps; these lesions most likely represent a response to a low-grade inflammatory stimulus. Such reactive foci should be designated as condensing osteitis (CO) or focal chronic sclerosing osteomyelitis, and should not be included under the designation of idiopathic osteosclerosis (1-2).

A review of available literature revealed that studies examining IO and CO lesions comparatively are quite limited (1). Furthermore, we identified no frequency studies carried out in this field in the Turkish population. The aim of this unique study for the study region with the largest sample size in the literature was to investigate the frequency of IO and CO lesions in the Turkish patient population with respect to age, sex, shape, localization, and dental relationship.

\section{Material and Methods}

We designed a descriptive study composed of 6,154 panoramic radiographs of patients who presented to our Oral and Maxillofacial Radiology Service of Dentistry Faculty, Ataturk University, between January 1996 and January 2008. All radiographs were performed using an orthopantomography device (Planmeca Proline CC 2002, 60-80 kVp, 8-10 mA, 12.8 second exposure time, Helsinki, Finland) by an X-ray technician who had a minimum working experience of 5 years as of 1996 . Radiographs were taken using 6-inch green-sensitive panoramic film (Medical, Konica Co., Japan) and an appropriate cassette-intensifying screen $(15 \times 30 \mathrm{~cm}$, Panoramic X-Ray Film Cassette, Planmeca, Finland-Lanex Screen, Kodak Eastman Co., USA). During filming, exposure adjustments were made in accordance with each individual and the films were developed using an automatic film-processing machine (Velopex, Extra-X, England).

To minimize variability in the present study, examinations were carried out jointly by the first 2 authors of the article (1 academic from the department of oral diagnosis and radiology and 1 academic from oral and maxillofacial surgery) over approximately 2 months. Throughout the study, in case of disagreement between the first 2 authors as to the outcome of an examination, the third author was consulted. Cases in which there was no consensus were excluded from the study.

For each panoramic film examined, the patient's age and sex was recorded as based on his/her patient file. Of the examined films, those diagnosed as showing IO or $\mathrm{CO}$ were scanned and digitized and then examinations were repeated. In addition, in patients identified as IO or $\mathrm{CO}$, the shape, localization, and dental relationships of the lesions were also recorded.

Lesions were considered to be $\mathrm{CO}$ if it was likely that they were associated with chronic inflammation. Generally, these lesions surrounded the apex of teeth with deep caries or large restorations. Lesions that did not show any obvious connection to inflammation, that were well demarcated, and were not ruled out by the instances listed above were identified as IO. The following radiopacities were specifically excluded (3-5):

- Characteristic mixed radiopaque-radiolucent areas of periapical cemental dysplasia and other benign fibro-osseous lesions of periodontal ligament origin (i.e., diffuse sclerosing osteomyelitis and florid osseous dysplasia).

- Increased thickening of the lamina dura around teeth that showed marked malposition or that served as abutment for fixed bridges or partial dentures.

- Clearly identifiable remnants of deciduous or permanent teeth.

- Radiopacities interpreted as tori or exostoses.

- Solitary radiopacities seen in edentulous regions, since these might have been excessively ossified surgical sites.

- Patients with Gardner's syndrome, familial polyposis of the colon, and other diseases with bone metabolic disturbance.

The shape of the lesions was classified as either round or irregular.

The location of the lesion was classified first as mandibular or maxillary, then further by region of the jaw: incisive, canine, canine-premolar, premolar, premolarmolar, or molar.

The relationship to teeth was defined using the criteria from Geist and Katz (5): (1) apical if the masses were predominately located around the root apices (Figs. 1A and 2A-B), (2) separate if the radiopacities were apical to and clearly separated from the teeth and lamina dura (Figs. 1B and 2A) (3) interradicular if the sclerotic tissue was limited to the area between the roots (Fig. 1C), and (4) apical and interradicular if the radiopacities were located at the apices and exhibited significant extension between the roots (Figs. 1D and 2C).

The variables were analyzed using the Statistical Package for Social Sciences (SPSS 11.5; Chicago, IL, USA) Program. The Chi-squared test was used to determine potential differences in the distribution of lesions when stratified by gender, age, number, shape, localization, and relation to teeth. A $p$ value of $<0.05$ was considered statistically significant. 

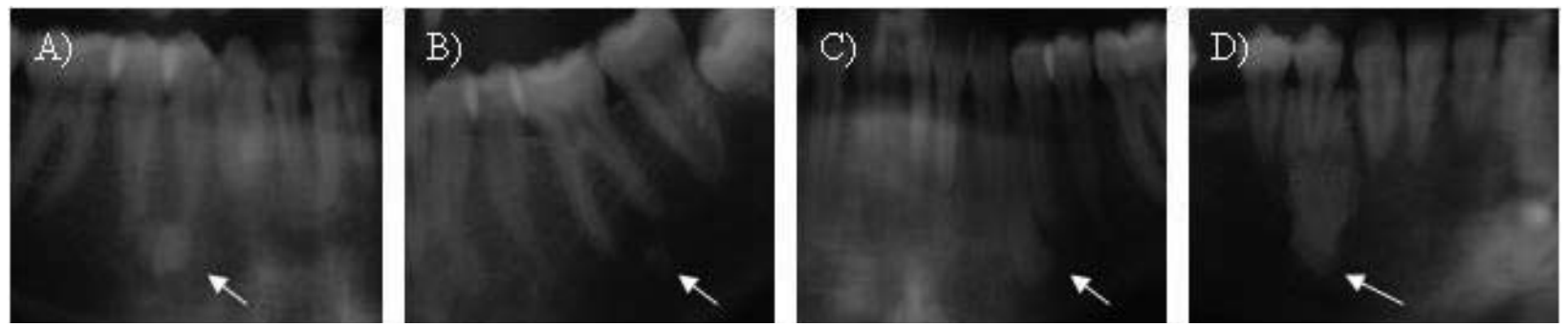

Fig. 1. IO lesions in terms of dental relationships.
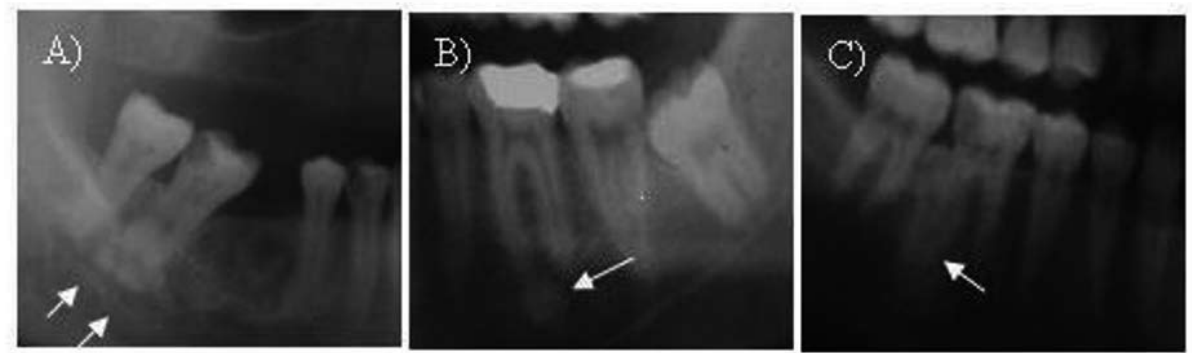

Fig. 2. CO lesions in terms of dental relationships.

\section{Results}

This study was performed on panoramic radiographies of 6,154 patients (2911 female, 3243 male), with different dental complaints, aged $5 \neg-69$ years (mean age: 33.4). There were 238 radiopacities detected, 185 IO lesions in $150(2.44 \%)$ subjects, and $53 \mathrm{CO}$ lesions in 50 $(0.81 \%)$ subjects. Of the subjects with multiple lesions, 23 had 2 IO, 3 had 2 CO, and 6 had 3 IO. No patient had IO and CO lesions concurrently. Both IO (96 female, 54 male) and CO (27 female, 23 male) lesions were found to be higher in number among females when compared to males. However, this difference was statistically significant only for IO lesions $(p<0.001)$. The number of IO lesions was found to be significantly higher in the $3 \mathrm{rd}$ and 4 th decades in life (20-39 years) than in other periods $(p<0.001)$. On the other hand, the frequency of $\mathrm{CO}$ lesions was the similar in the age ranges of 20-39 years and 40-69 years, and its frequency in these periods was statistically higher than in the 1st and 2nd decades of life $(\mathrm{p}<0.01)$ (Table 1 and 2).

Table 1. Patients with idiopathic osteosclerosis lesion and selected covariates: sample size and frequency.

\begin{tabular}{|l|l|l|c|c|c|c|}
\hline & & $\mathbf{n}$ & Patients with IO Lesions & $\mathbf{\%}$ & $\mathbf{X}^{2}$ & P value \\
\hline \multirow{3}{*}{ Gender } & Female & 2911 & 96 & 3.3 & \multirow{2}{*}{17.20} & \multirow{2}{*}{0.0001} \\
\cline { 2 - 7 } Age Groups & Male & 3243 & 54 & 1.7 & & \\
\hline & $5-19$ years & 1969 & 17 & 0.9 & & \\
\cline { 2 - 7 } & $20-39$ years & 2619 & 123 & 4.7 & \multirow{3}{*}{98.03} & \multirow{2}{*}{0.0001} \\
\cline { 2 - 6 } & $40-69$ years & 1566 & 10 & 0.6 & & \\
\hline
\end{tabular}

Table 2. Patients with condensing osteitis lesions and selected covariates: sample size and frequency.

\begin{tabular}{|c|c|c|c|c|c|c|}
\hline & & $\mathbf{n}$ & Patients with CO Lesions & $\mathbf{\%}$ & $\mathbf{X}^{\mathbf{2}}$ & P value \\
\hline \multirow{3}{*}{ Gender } & Female & 2911 & 27 & 0.9 & \multirow{2}{*}{0.91} & \multirow{2}{*}{0.34} \\
\cline { 2 - 7 } & Male & 3243 & 23 & 0.7 & & \\
\hline \multirow{3}{*}{ Age Groups } & $5-19$ years & 1969 & 5 & 0.3 & & \multirow{2}{*}{0.003} \\
\cline { 2 - 7 } & $20-39$ years & 2619 & 27 & 1.38 & \multirow{2}{*}{0.1} \\
\cline { 2 - 7 } & $40-69$ years & 1566 & 18 & & \\
\hline
\end{tabular}


Table 3. The distributions of idiopathic osteosclerosis and condensing osteitis lesions with respect to shape, localization, and dental relationship.

\begin{tabular}{|c|c|c|c|c|c|c|c|}
\hline & & $\begin{array}{l}\text { (184 IO lesions } \\
\text { in } 149 \text { subjects) }\end{array}$ & $\%$ & $\begin{array}{l}\text { (54 CO lesions } \\
\text { in } 51 \text { subjects) }\end{array}$ & $\%$ & $\mathbf{X}^{2}$ & $P$ value \\
\hline \multirow{2}{*}{ Shape of lesions } & Round & 127 & 68.6 & 37 & 69.8 & \multirow{2}{*}{0.03} & \multirow{2}{*}{0.87} \\
\hline & Irregular & 58 & 31.4 & 16 & 30.2 & & \\
\hline \multirow{2}{*}{ Localization of lesions } & Mandible & 183 & 98.9 & 53 & 100.0 & \multirow{2}{*}{ - } & \multirow{2}{*}{$1.0^{*}$} \\
\hline & Maxilla & 2 & 1.1 & 0 & 0.0 & & \\
\hline \multirow{6}{*}{$\begin{array}{c}\text { Regional localization } \\
\text { of lesions }\end{array}$} & Incisive & 2 & 1.1 & 0 & 0.0 & \multirow{6}{*}{42.71} & \multirow{6}{*}{0.0001} \\
\hline & Canine & 12 & 6.5 & 0 & 0.0 & & \\
\hline & $\begin{array}{l}\text { Canine-Pre- } \\
\text { molar }\end{array}$ & 18 & 9.7 & 0 & 0.0 & & \\
\hline & Premolar & 55 & 29.7 & 2 & 3.8 & & \\
\hline & $\begin{array}{l}\text { Premolar- } \\
\text { Molar }\end{array}$ & 13 & 7.0 & 0 & 0.0 & & \\
\hline & Molar & 85 & 46.0 & 51 & 96.2 & & \\
\hline \multirow{4}{*}{$\begin{array}{l}\text { The relationship to } \\
\text { teeth of lesions }\end{array}$} & Apical & 55 & 29.7 & 31 & 58.5 & \multirow{4}{*}{57.11} & \multirow{4}{*}{0.0001} \\
\hline & Interradicular & 35 & 18.9 & 0 & 0.0 & & \\
\hline & $\begin{array}{c}\text { Apical and } \\
\text { interradicular }\end{array}$ & 21 & 11.4 & 21 & 39.5 & & \\
\hline & Separate & 74 & 40.0 & 1 & 2.0 & & \\
\hline
\end{tabular}

Lesions had an almost equal distribution in terms of shape. Both types of lesions were determined to be round (nearly $70.0 \%$ ) or irregular (30.0\%) in shape. Similarly, all CO lesions and $98.9 \%$ of the IO lesions were located in the mandible ( $>0.05)$. Only 2 IO lesions were found in the upper jaw. The lesions largely involved the premolar and molar regions. However, their comparison revealed statistical differences. While IO lesions exhibited a higher rate of involvement in the premolar region than $\mathrm{CO}$ lesions, molar region involvement by $\mathrm{CO}$ lesions was higher than that of IO lesions $(p<0.001)$. Such significant differences were also found with regard to a lesion's relationship to teeth. Occurring in the dental apical and apical+interradicular regions, the $\mathrm{CO}$ lesions were greater in number than the IO lesions. In contrast, the type of dental relationship in which IO lesions were more frequent than $\mathrm{CO}$ lesions was determined as a separate dental relationship (Table 3).

\section{Discussion}

The prevalence of radiopaque lesions as presented in the literature ranges from $3.3 \%$ to $31.0 \%(6-8)$. Geist and Katz (5) observed 5.4\% frequency of idiopathic osteosclerosis in 1,921 full-mouth intraoral radiographic surveys. The idiopathic osteosclerosis frequency of $6.1 \%$ on 1,047 panoramic radiographs of patients by Yonetsu et al. (4) was approximately two thirds, as high as previously reported by Kawai et al. (9) on panoramic radiographs of 1,203 patients. On the other hand, El- liasson et al. (10) observed a $2 \%$ prevalence of condensing osteitis in a study of periapical radiographs of 1,149 roots and their files, whereas $6 \%$ of the periapical radiographs were recorded to have condensing osteitis in a smaller study (11) of 889 patients. The present examination found IO prevalence to be $2.4 \%$ and a lower CO prevalence at $0.8 \%$. To our knowledge, this study was the first to examine the prevalence of radiopacities in the jawbones of the Turkish population, with the largest sample size used to date. The low frequency found in the present examination might first be attributed to the exclusive use of panoramic radiographs to identify the lesions. This could be evidenced by higher prevalence rates due to the use of periapical radiographs $(5,11,12)$. A second reason might be the differences among the descriptive criteria used in the studies. For instance, we excluded radiopacities occurring in edentulous regions, as these could be excessively ossified regions that formed after surgery. Yet, these regions might also be areas of condensing osteitis that remained after tooth extraction. As another reason, genetic variations and differences in diet might also contribute to this low rate.

In the present study, both IO and CO lesions had a higher prevalence among women than among men. This finding agrees with Geist and Katz (5) and McDonnell (13), who found a female to male ratio of $1.5: 1$ and $2: 1$, respectively. Similarly, Avramidou et al. (14) detected that female patients were more likely to have a radiopaque lesion than men. However, Kawai et al. (9) and Yonetsu 
et al. (4) found no difference between the incidence in women and men.

Kawai et al. (9) found a marked decrease with advanced age in the prevalence of periapical enostosis (idiopathic osteosclerosis related to teeth), while they also observed a sharp increase in solitary enostosis (idiopathic osteosclerosis not related to teeth) during the seventh decade, which they attributed to the automatic replacement of periapical enostosis by solitary enostosis. In the present study, both IO and CO lesions were found in the 3rd and 4th decades of life with a marked frequency. In addition, $\mathrm{CO}$ lesions were observed with the similar frequency in the age ranges of 40 to 69 years and 20 to 39 years. A possible explanation might be increased tooth caries and pulpal infections with increased age. The high prevalence of IO lesions in the 3rd and 4th decades coincides with the maximum bone mass acquisition in these periods. Petrikowski and Peters (3), in their follow-up study on orthodontic patients with IO lesions and a mean age of 14 years, found that lesions are apt to change and grow, particularly during adolescence; on the other hand, Williams and Brooks (1) observed minimal changes in lesions in the follow up of a series with a mean age of 44 years.

A higher prevalence of radiopacities was found in the mandible than in the maxilla. This might partly be explained by the fact that when panoramic radiographs are examined and assessed, there are fewer problems with the superimposition of anatomic structures in the mandible than in the maxilla. In addition, it may be attributed to differences in bone anatomy and blood supply. By definition, the cause of $\mathrm{IO}$ and $\mathrm{CO}$ lesions is obscure. In the statistical evaluation in our study, IO lesions were more frequent in the premolar region than $\mathrm{CO}$ lesions, while $\mathrm{CO}$ lesions were observed to be more frequent in the molar region. It has been maintained that since IO lesions are most frequently found located in the premolar and molar areas, they might represent residual roots from deciduous molars, resorbed and replaced by sclerotic bone (15). Histological examination in the study of Henrikson et al. (16) clearly demonstrated sclerotic bone containing a retained root in one case. So, it is possible that microscopic root fragments may act as a nidus for bone proliferation in some cases. On the other hand, the frequency of $\mathrm{CO}$ lesions in the molar region could be attributed to caries, traumatic occlusal stress, and pulpal infections, which occur more frequently in molar teeth than in premolar teeth. In addition, nearly $40 \%$ of the IO lesions in our examination were separate lesions unrelated to the region, upon which neither residual root fragments nor excessive occlusal forces can have a stimulator effect. CO lesions, on the other hand, were observed to exhibit mostly apical and apical+interradicular involvement $(58.5+39.5=98.0 \%)$. Therefore, while IO lesions are developmental varia- tions of normal bone architecture unrelated to a local stimulant, $\mathrm{CO}$ lesions could be considered reactive formations related to teeth with severe caries, restoration, or pulpitis.

In differential diagnosis of $\mathrm{IO}$ and $\mathrm{CO}$ lesions, root segments, exostoses, foreign bodies, or even impacted teeth should be taken into consideration (14). Extraction history and the lines of a residual root fragment facilitate the distinction with root segments. The complete and accurate shape of impacted teeth is sufficient to confirm diagnosis. Exostoses are single or multiple radiopaque lesions of the periosteal surface of the jaw; they appear radiographically as clearly outlined radiopacities.

IO lesions is usually asymptomatic, but it may cause changes in tooth position or problems during orthodontic treatment $(4,13)$. Marques Silva et al. (17) presented report a case of tooth resorption caused by ectopic eruption rote induced IO.

In conclusion, this study has been the first to examine the prevalence of these lesions in the Turkish population, with the largest sample size in the literature. Furthermore, it has been one of the few studies comparatively examining IO and CO lesions. Our findings demonstrate that these lesions have a lower prevalence in the Turkish population and are more frequent among women, in particular age decades, and in the mandibular premolar-molar region.

\section{References}

1. Williams TP, Brooks SL. A longitudinal study of idiopathic osteosclerosis and condensing osteitis. Dentomaxillofac Radiol. 1998;27:275-8.

2. MacDonald-Jankowski DS. Idiopathic osteosclerosis in the jaws of Britons and of the Hong Kong Chinese: radiology and systematic review. Dentomaxillofac Radiol. 1999;28:357-63.

3. Petrikowski CG, Peters E. Longitudinal radiographic assessment of dense bone islands of the jaws. Oral Surg Oral Med Oral Pathol Oral Radiol Endod. 1997;83:627-34.

4. Yonetsu K, Yuasa K, Kanda S. Idiopathic osteosclerosis of the jaws: panoramic radiographic and computed tomographic findings. Oral Surg Oral Med Oral Pathol Oral Radiol Endod. 1997;83:517-21.

5. Geist JR, Katz JO. The frequency and distribution of idiopathic osteosclerosis. Oral Surg Oral Med Oral Pathol. 1990;69:388-93.

6. Farman AG, De V Joubert JJ, Nortjé CJ. Focal osteosclerosis and apical periodontal pathoses in "European" and Cape coloured dental outpatients. Int J Oral Surg. 1978;7:549-57.

7. Alattar MM, Baughman RA, Collett WK. A survey of panoramic radiographs for evaluation of normal and pathologic findings. Oral Surg Oral Med Oral Pathol. 1980;50:472-8.

8. Austin BW, Moule AJ. A comparative study of the prevalence of mandibular osteosclerosis in patients of Asiatic and Caucasian origin. Aust Dent J. 1984;29:36-43.

9. Kawai T, Hirakuma H, Murakami S, Fuchihata H. Radiographic investigation of idiopathic osteosclerosis of the jaws in Japanese dental outpatients. Oral Surg Oral Med Oral Pathol. 1992;74:237-42.

10. Eliasson S, Halvarsson C, Ljungheimer C. Periapical condensing osteitis and endodontic treatment. Oral Surg Oral Med Oral Pathol. 1984;57:195-9.

11. Marmary Y, Kutiner G. A radiographic survey of periapical jawbone lesions. Oral Surg Oral Med Oral Pathol. 1986;61:405-8.

12. Rohlin M, Kullendorff B, Ahlqwist M, Henrikson CO, Hollender L, Stenström B. Comparison between panoramic and periapical radi- 
ography in the diagnosis of periapical bone lesions. Dentomaxillofac Radiol. 1989;18:151-5.

13. McDonnell D. Dense bone island. A review of 107 patients. Oral Surg Oral Med Oral Pathol. 1993;76:124-8.

14. Avramidou FM, Markou E, Lambrianidis T. Cross-sectional study of the radiographic appearance of radiopaque lesions of the jawbones in a sample of Greek dental patients. Oral Surg Oral Med Oral Pathol Oral Radiol Endod. 2008;106:e38-43.

15. Gibilisco JA. Stafne's Oral Radiographic Diagnosis. 5th edn. Philadelphia. USA: W.B. Saunders Company; 1985. p. 142-6.

16. Henrikson CO, Nordenram A, Nyborg H. Radiopaque areas in human jaws. A report of 18 cases. Scand J Dent Res. 1963;71:373-9. 17. Marques Silva L, Guimaraes AL, Dilascio ML, Castro WH, Gomez RS. A rare complication of idiopathic osteosclerosis. Med Oral Patol Oral Cir Bucal. 2007;12:E233-4. 